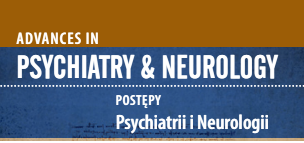

Correspondence to:

Izabela Oleksak

Department of Applied Psychology,

Medical University of Lublin, Poland

ul. Chodźki 7

20-093 Lublin, Polska

e-mail: 54911@student.umlub.pl

Submitted: 02.06.2020

Accepted: 22.08.2020

\section{PHANTOM OUTSIDE THE OPERA - A REVIEW OF CURRENT FINDINGS ON TREATING PHANTOM PAIN}

\author{
Izabela Oleksak, Dominika Psiuk, Karolina Maliszewska, \\ Agnieszka Kaczmarska
}

Department of Applied Psychology, Medical University of Lublin, Poland

\begin{abstract}
Purpose: Presentation of the latest discoveries and progress in treating phantom limb pain (PLP).

Views: A variety of strategies are used in PLP therapy. Among non-pharmacological methods the best results are obtained by mirror therapy, which creates the illusion of an existing healthy limb. Other advantages of this therapy are its non-invasive character and low cost. Other methods of which much is expected are: graded motor imagery (GMI), hypnosis, electromagnetically shielding limb liner, and virtual reality. Many drugs are taken into consideration as potential agents that may lower the pain, including nonsteroidal anti-inflammatory drugs (NSAIDs) and acetaminophen, opioids, antidepressants, anticonvulsants, etc. Although nowadays nonsteroidal anti-inflammatory drugs are most commonly used, researches are mostly focused on gabapentin, which shows efficacy. From a psychological point of view, it is important to remember that following amputation patients experience grief for the loss of the prior body image. Many factors pre-dating surgery contribute to difficulties in undergoing such a process, as well as a feeling of increasing pain, so it is worth including teams of psychologists who take care of patients before and after amputation.

Conclusions: Current attempts to treat PLP, show differing degrees of efficacy among patients, so it is therefore impossible to set a consistent treatment scheme. For this reason alone, it is recommended to select appropriate therapy on an individual basis. Knowing the precise pathophysiology of an individual's pain could be helpful in improving the treatment methods. The character of pain of this sort suggests the necessity of engaging specialists from different fields in order to treat the condition.
\end{abstract}

Key words: pain treatment, amputation, phantom limb pain.

\section{INTRODUCTION}

Phantom limb pain (PLP) is a chronic condition, occurring in $45-85 \%$ of patients following amputation surgery. It consists of a painful, debilitating sensation which affects both physical and mental health [1]. The aetiology of PLP is as yet unknown, however, there are some theories about its mechanisms, such as an underlying psychiatric illness, the formation of neuroma and changes in the peripheral nervous system [2]. As our understanding of the condition's mechanisms is still incomplete, it is difficult to adjust prevention or find proper treatment aligned with the specific needs of post-amputation patients [3]. The purpose of this work is to review the recent literature on PLP therapies and treatment.

\section{STATE OF KNOWLEDGE}

Managing phantom pain requires a patient-centred approach, so it is difficult to propose one correct treatment plan for all patients. While treating a patient with an amputated limb, one must be aware that pain is not the only thing with which they struggle. Mental attitude and fear of pain can significantly enhance the experience. Therapeutic success depends not only on pharmacotherapy, but also on rehabilitation techniques, such as transcutaneous nerve stimulation (TENS), mirror therapy, transcranial magnetic stimulation, spinal cord stimulation, acupuncture and even hypnosis.

Mirroring is gaining more and more supporters among doctors. In this method, the patient places himself or herself in front of a mirror in such a way that the whole healthy limb is reflected instead of the stump. Using the voluntary movements of the healthy limb, the mirror creates the illusion that the patient's amputated limb is still capable of painless movement. The patient's brain is cheated. As a result of the stimulation delivered, the perception of the body changes. 
The projections are received in corresponding motor and sensory cortical pathways, which reduces the risk of damage tied to receiving sensory information. It has been proved that after amputation, the connections of the primary sensory and motor cortical areas with the amputated limb were no longer necessary, as those areas were replaced by adjacent cortical areas. The degree of some sort of reorganization of the cortex after amputation may be correlated with the intensity of pain $[4,5]$.

American scientists checked whether every case of PLP responds to mirror therapy. They also wondered if a potential response could be related to the intensity of pain in a moment of including the patient in the study. At the beginning of the study, patients after a onesided amputation of the lower limb were divided into two groups. Their pain level was measured using a visual analogue scale and the McGill Pain Short-Form Questionnaire, and then the subjects underwent 15-minute mirror therapy sessions. It was proved that it was possible to predict how long the therapy would last depending on the baseline PLP result. In patients with low PLP, pain relief was achieved after 7 treatment sessions, with a median of 14 and a high after 21 sessions [7]. Another study compared the effectiveness of mirror therapy with percutaneous nerve stimulation (PNS). Results showed that although there was a significant reduction in pain, it was not clear, which of the therapies was more effective [8]. There are disagreements over the way in which the therapy should be conducted. Some say that the limb should be fully reflected in the mirror, while others maintain that it is enough if only part of it is reflected. Many differences are also observed regarding the duration of a single session (from 15-40 minutes or until muscle fatigue) as well as the duration of the entire therapy (from several days to a few months). Some authors also suggest starting therapy two weeks before amputation if it is a scheduled surgery. There is also a question of whether the illusion that the missing limb is intact might be an obstacle in choosing a properly selected prosthetic limb. As a result of mirror therapy, belief in a full recovery may be so deep that the patient refuses further treatment.

Mirror therapy is a promising method for treating phantom limb pain. It is increasingly recommended as an adjunct to phantom pain treatment. This method meets the WHO's recommendations; among its advantages are much lower cost than that of other therapies, easy accessibility and the possibility of doing the exercises without the help of qualified personnel [6]. However, scientists note that most studies are conducted on a small number of patients, and that they differ in terms of method, duration and the criteria for including patients. Therefore, scientists assert that there is a need to conduct further studies on large groups of patients to confirm the effectiveness of this therapy.

\section{GRADED MOTOR IMAGERY}

This is a technique that consists of three stages. In the first stage, pictures of limbs are shown to patients and they have to decide whether they see the right or the left limb. During the second stage, patients are told to close their eyes and imagine performing a specified move. The exercise needs to be done three times a day for about 15 minutes. The third stage is mirror therapy, as previously described.

During 6-week graded motor imagery (GMI) programme, patients who received GMI were compared to those who received routine physiotherapy. The comparison focused on the level of reduction of PLP. Studies have shown that in comparison to normal physiotherapy, GMI reduced phantom pain to a higher degree and the effect of reduced PLP persisted for half a year, though no statistically significant improvement was observed in the quality of patients' life $[9,10]$. Even so, GMI therapy provides many benefits. First of all, it is low-cost and therefore can be used by most patients. The treatment is non-invasive, and its side effects are minimal. All these arguments speak in favour of including GMI therapy in routine clinical practice for patients with PLP [11].

\section{HYPNOSIS}

Hypnosis is a state of mind in which a person shows greater suggestibility, has only selective capability of focus and has limited consciousness of perceived external stimuli [12]. It has been noted that hypnosis can change the perception of particular experiences and has therefore been used in pain therapy. Studies show that phantom pain can be reduced with the use of hypnosis. The somatosensory cortex and anterior cingulated cortex modify pain perception. It seems that hypnosis acts on those brain areas and changes the perception of pain stimuli. Hypnosis mostly leads to activation of the brain area rather than its deactivation. Moreover, activation of the prefrontal cortex during therapy provides positive imaginative experiences and induces hypno-anaesthesia, i.e. is a state of mind in which a patient feels no pain while the therapy is being administered [13]. Hypnosis produces positive effects regardless of the cause of amputation (injury, vascular problems). To carry out experiments, hypnotic techniques were matched individually to each patient. It was proved that patients' pain decreased significantly after only four sessions. It is not clear how long the desired effect lasts following the end of therapy. Interestingly, thanks to hypnosis the dozes of analgesic medication taken by patients could be reduced [14]. These studies encouraged the use of hypnosis in modern clinical practice. 


\section{ELECTROMAGNETICALLY SHIELDING LIMB LINER}

During therapy a special inset is put between the stump and the prosthesis. It is made of material of great electrical conductivity, which protects a particular body area from the influence of the electromagnetic field coming from the outside. Studies have been conducted, in which the effects of electromagnetically (EM) shielding and functioning of the inset without conductivity were compared. The patients chosen for the study had experienced persistent pain during the day whose intensity fluctuated. Studies showed that EM shielding caused significant decrease of maximal pain felt by patients. Besides, patients who underwent therapy had better health than the placebo group. The mechanism explaining the reason for reducing patients' pain symptoms is not entirely known. It is possible that the limitation of nerve endings' activity at the spot of the amputated limb is responsible. EM shielding, with the help of the electromagnetic field created, probably limits the flow of calcium ions to the nerve cells, thereby minimizing the emergence of impulses that generate pain sensations [15]. Subsequent studies reported decreases in both maximal and medium phantom pain and improvement of mood among EM-shielding patients. Not all of the results of the studies involving this kind of therapy are entirely clear. Some of them prove that EM shielding is really effective after short-term use, whilst others do not show statistically significant reduction of PLP after 12-week course of therapy [16]. Therefore, it is necessary to ensure further studies to improve EM shielding therapy and its widespread use among patients with PLP.

\section{VIRTUAL REALITY}

One of the latest methods for helping patients cope with PLP takes advantage of virtual reality (VR) therapy. It starts with placing sensors on the patient's stump. The next step is myoelectrical registration which converts commands sent to the muscles into the image being seen with the help of VR glasses. Patients watch the image of their well-functioning limbs while (mostly) playing in physically engaging games. The whole process allows patients to feel a restoration of feeling of an intact limb and full control of their own body. A significant decrease in intensity of pain was shown directly after each session; during one session of this kind the pain completely subsided. Moreover, it was shown that during therapy and directly before each session the level of pain was progressively lower [17]. Subsequent studies also showed promising results. After the first session with the use of VR technique most of the participants did not experience pain at all. They also gave good reviews on realism, treatment satisfaction and entertainment [18]. The pre- eminent advantage of this method is the sense of safety it provides linked to the fact that the patient does not feel pain after making a wrong "move" and can focus more on the task [19]. Some studies compare this method with mirror therapy, though it is not clear which is more effective. Perhaps mirror therapy is more likely to be used because of its lower costs. Further investigation is needed to systematize this issue [20].

There is still scope for new and more effective agents in PLP treatment. In spite of a wide range of pharmacological treatments available, it remains unclear which is most reliable [21]. Nowadays, the most commonly used medication are nonsteroidal anti-inflammatory drugs (NSAIDs) and acetaminophen, opioids, antidepressants, anticonvulsants, $N$-methyl-D-aspartate receptor (NMDAR) antagonists and calcitonin [22]. Botulinum neurotoxins and local anaesthetics have also been examined in view of their effectiveness in PLP [23].

\section{ANALGESICS}

NSAIDs are most common in the treatment of PLP, but their efficacy is not sufficient. They are also one of the least studied agents [22]. Opioids, on the other hand, have shown efficacy in PLP treatment. Morphine, tramadol and methadone were mainly studied, and demonstrated effectiveness $[21,23,24]$. A case was reported of a patient with an above-knee amputation, who was given morphine orally. The initial dose was low and resulted in total pain relief. After a week without morphine, symptoms of phantom pain appeared, so the dose had to be increased (from initial $3 \mathrm{mg}$ twice per day to $540 \mathrm{mg}$ every four hour. Any attempt to withdraw morphine caused renewal of the pain. The patient did not show any signs of toxicity or opioid dependence [25].

\section{ANTIDEPRESSANTS}

Amitryptyline and venlafaxine are commonly used in PLP patients. However, there is very little evidence of their effectiveness. Out of the two randomized studies of amitryptyline efficacy, one failed to find any differences between groups taking amitryptyline and the placebo [26]. The second study demonstrated amitryptyline efficacy [27]. Other antidepressants, for example, duloxetine, venlafaxine, chlorimipramine or nortriptyline are also used [23].

\section{ANTICONVULSANTS}

Nowadays, gabapentin is the most studied agent as its efficacy has been proven in a number of studies. One of the recent randomized studies showed that postoperative pain intensity was lower in the gabapentin group compared to placebo in paediatric patients with amputation 
linked to malignant bone tumours [28]. Pregabaline and valproic acid (VPA) have been, among others, most recently studied anticonvulsants. In a case report discussing a 4-year-old girl after a forefoot amputation, she received pregabaline in addition to analgesics because the initial pain therapy had not been sufficient. This multimodal treatment showed high effectiveness, as the pregabaline demonstrated pain and anxiety reduction in the patient [29]. A randomized study of the efficacy of valproic acid in post-amputation pain prevention showed no differences between the VPA and placebo group, in terms of effectiveness or adverse events [30].

\section{NMDAR ANTAGONISTS}

Ketamine was studied in connection with phantom pain treatment and demonstrated efficacy in some cases, but produced substantial side effects, including cystitis, hallucination or cardiovascular effects. Other NMDAR antagonists studied have been dextromethorphan and memantine $[21,31]$.

\section{CALCITONIN}

There are two recent studies about a calcitonin impact in PLP. In one randomized study patients were scheduled for lower limb amputation and received calcitonin with bupivacaine and fentanyl or bupivacaine and fentanyl alone in the pre- and postoperative periods. The study showed that phantom pain remained at grade I (mild paraesthesias not interfering with everyday activities) in the calcitonin group. Pain was measured one month, three months, six months and one year after amputation, and in the calcitonin group respectively $96.7 \%, 93.3 \%$, $90 \%$ and $87.6 \%$ patients remained at grade I, and after a year only 4 patients progressed to II (uncomfortable paraesthesias, not interfering with activities) or III (intense, frequent pain). On the other hand, in the bupivacaine-fentanyl group $90 \%, 80 \%, 60 \%$ and $50 \%$ patients respectively remained at grade I during one-year follow up, and one year after amputation 15 patients progressed to grades II or III [32]. There was a case report of a patient with left forearm amputation. After the surgery, neither non-pharmacological nor first-line pharmacological treatment was effective, due to a comorbid complication with heterotopic ossification. The patient started receiving 200 units of calcitonin intranasally daily and after one month reported a reduction in the frequency and intensity of phantom limb pain [33].

\section{BOTULINUM TOXIN}

A minor clinical observation demonstrated a notable impact of botulinum neurotoxin in phantom limb pain [34], however, a randomized study was recently conducted and did not show a difference in phantom pain reduction between the botulin and placebo groups. On the other hand, the study demonstrated significant sweat reduction and improved prosthetic function in the botulin group as compared to the placebo group [35].

\section{LOCAL ANAETHETICS}

Previous studies examined bupivacaine and lidocaine and showed that bupivacaine reduced phantom sensations, while lidocaine did not [23]. A recent clinical trial of lidocaine's impact on lower limb amputation pain was cancelled due to lack of recruitment [23].

Limb amputation, followed by disability, is almost always considered as the most radical way to save life or health endangered by diabetes, arteriosclerosis or injury, among other reasons. Fitting prostheses and teaching patients how to function with them should solve the problem, yet this is not entirely true because many factors contribute to the presence of residual limb pain (RLP) or PLP which play a substantial role in the process of adaptation to this new situation.

\section{PROGNOSTIC FACTORS}

It is crucial to understand these before preparing for the occurrence of PLP. Among the so-called positive factors, which suggest the possibility of increased pain, are advanced age, above-knee amputation, intensity of phantom limb sensation (PLS), pain before amputation, greater level of pre-amputation anxiety and depression $[36,37]$. Feeling of movement and response to earlier treatment are connected with lower level of pain (those are negative factors). Particular attention was paid in one study to the age factor because ageing results in neural pathways adaptation difficulties which decrease ability to cope with chronic pain [36]. A different study showed a connection between PLP and PTSD with the higher possibility of PLP emerging with the higher severity PTSD [38]. Other researchers found that if pain before amputation persisted for at least a month, PLP emerged more frequently [39].

\section{CAUSES}

Many studies try to explain the PLP phenomenon. The theory of homunculus can be helpful, according to which every part of the body has its own representation in the cortex. Even though it may suggest that the amputation stops the connection, it does not happen. Despite being cut-off from stimuli and the reorganization of the neural pathways that follows, the limb is still strongly represented [40]. The process reorganization is described as "maladaptive plasticity" and is believed to be the basis of the occurrence and persistence of PLP [41, 42]. A similar theory is that of "proprioceptive memory", 
which assumes that in every man's mind there is an awareness of the existence of limbs, as well as a memory of their localization and position. This memory remains even after amputation [43].

\section{PATIENTS’ EXPERIENCE}

While working with patients, it is important to bear in mind that amputation does not only mean the lack of a limb, but also partial destruction of their world and future. From a psychological point of view, this situation brings out their most primary anxieties so the whole process could be called "grief after loss of body image", which consists of five well-known psychological stages: denial, anger, bargaining, depression and acceptance [44]. Body image incorporates emotions matched to factors that define satisfaction with one's own appearance (it can relate to the whole body or its parts) [45]. Therefore, unsurprisingly, patients avoid situations which remind them of their condition and prefer to focus on memories about the healthy limb - whereby conviction about the intact "phantom limb" intensifies [44]. The statement of a patient experiencing pain after amputation shows that a clear image of a phantom limb was considered by her as a positive feeling [46]. Another study showed an interesting fact that patients who experienced PLP tended to plan and imagine the movement of the missing limb with high precision. This was considered an argument for treating PLP as a body image distortion [47]. There are other coping strategies applied by patients. Among a few mentioned in various studies are distraction, relaxation, seeking support, exercise, manipulation of the residual limb and drug or alcohol abuse. None of them helped reduce PLP [48] which also suggests that emotions felt by patients during different phases of treatment should also be treated as important by doctors and psychologists. A study showed that the differences between people who experience PLP and those who do not concern only depression and anxiety, which suggests the lack of connection between PLP and psychiatric symptoms. Levels of those two conditions initially tend to decrease after rehabilitation, but are high again two or three years later. Simultaneously, it was pointed out that there was no psychiatric examination conducted among patients and that the results should be confirmed on a greater number of patients [49].

Despite some limitations of research, psychotherapy has been proven to show good results in amputees [50], particularly when it focuses on changing the patient's perception and experience of pain. Its particular kinds, such as cognitive behavioral therapy or acceptance and commitment therapy, can even prevent PLP [51]. It is important to remember that the best treatment effects are achieved by teams consisting of specialists from different fields, including prosthetic professionals, mental health nurses, psychotherapists, pharmacists, clinicians and a pain specialists [52].

\section{Conflict of interest}

Absent.

Financial support

Absent

\section{References}

1. Kuffler DP. Origins of Phantom Pain. Mol Neurobiol 2018; 55: 60-69.

2. Poor Zamany Nejatkermany M, Modirian E, Soroush M, Masoumi M, Hosseini M. Phantom Limb Sensation (PLS) and Phantom Limb Pain (PLP) among Young Landmine Amputees. Iran J Child Neurol 2016; 10: 42-47.

3. Collins KL, Russell HG, Schumacher PJ, Robinson-Freeman KE, O'Conor EC, Gibney KD, et al. A review of current theories and treatments for phantom limb pain. J Clin Invest 2018; 128: 2168-2176.

4. Barbin J, Seetha V, Casillas JM, Paysant J, Pérennou D. The effects of mirror therapy on pain and motor control of phantom limb in amputees: a systematic review. Ann Phys Rehabil Med 2016; 59: 270-275.

5. Deconinck FJ, Smorenburg AR, Benham A, Ledebt A, Feltham MG, Savelsbergh GJ. Reflections on mirror therapy: a systematic review of the effect of mirror visual feedback on the brain. Neurorehabil Neural Repair 2015; 29: 349-361.

6. Wittkopf PG, Johnson MI. Mirror therapy: a potential intervention for pain management. Rev Assoc Med Bras 2017; 63: 1000-1005.

7. Griffin SC, Curran S, Chan AWY, Finn SB, Baker CI, Pasquina PF, et al. Trajectory of phantom limb pain relief using mirror therapy: Retrospective analysis of two studies. Scand J Pain 2017; 15: 98-103. 
8. Tilak M, Isaac SA, Fletcher J, Vasanthan LT, Subbaiah RS, Babu A, et al. Mirror therapy and transcutaneous electrical nerve stimulation for management of phantom limb pain in amputees - a single blinded randomized controlled trial. Physiother Res Int 2016; 21: 109-115.

9. Limakatso K, Madden VJ, Manie S, Parker R. The effectiveness of graded motor imagery for reducing phantom limb pain in amputees: a randomised controlled trial. Physiotherapy 2019; S0031-9406(18)30188-3.

10. Batsford S, Ryan CG, Martin DJ. Non-pharmacological conservative therapy for phantom limb pain: A systematic review of randomized controlled trials. Physiother Theory Pract 2017; 33: 173-183.

11. Limakatso K, Corten L, Parker R. The effects of graded motor imagery and its components on phantom limb pain and disability in upper and lower limb amputees: a systematic review protocol. Syst Rev 2016; 5: 145.

12. Ahlskog G. Clinical hypnosis today. Psychoanal Rev 2018; 105: 425-437.

13. Del Casale A, Ferracuti S, Rapinesi C, Serata D, Caltagirone SS, Savoja V, et al. Pain perception and hypnosis: findings from recent functional neuroimaging studies. Int J Clin Exp Hypn 2015; 63: 144-170.

14. Cárdenas K, Aranda M. Psychotherapies for the treatment of phantom limb pain. Rev Colomb Psiquiatr 2017; 46: 178-186.

15. Fisher K, Oliver S, Sedki I, Hanspal R. The effect of electromagnetic shielding on phantom limb pain: a placebo-controlled double-blind crossover trial. Prosthet Orthot Int 2016; 40: 350-356.

16. Ghoseiri K, Allami M, Soroush MR, Rastkhadiv MY. Assistive technologies for pain management in people with amputation: a literature review. Mil Med Res 2018; 5: 1.

17. Ambron E, Miller A, Kuchenbecker KJ, Buxbaum LJ, Coslett HB. Immersive low-cost virtual reality treatment for phantom limb pain: evidence from two cases. Front Neurol 2018; 9: 67.

18. Rutledge T, Velez D, Depp C, McQuaid JR, Wong G, Jones RCW, et al. A virtual reality intervention for the treatment of phantom limb pain: development and feasibility results. Pain Med 2019; 20: 2051-2059.

19. Stasieńko A, Sarzyńska-Długosz I. Virtual reality in neurorehabilitation. Postępy Rehabilitacji 2016; 4: 67-75.

20. Rothgangel A, Bekrater-Bodmann R. Mirror therapy versus augmented/virtual reality applications: towards a tailored mechanism-based treatment for phantom limb pain. Pain Manag 2019; 9: 151-159.

21. Hall N, Eldabe S. Phantom limb pain: a review of pharmacological management. Br J Pain 2018; 12: 202-207.

22. Luo Y, Anderson TA. Phantom limb pain: a review. Int Anesthesiol Clin 2016; 54: 121-139.

23. Alviar MJ, Hale T, Dungca M. Pharmacologic interventions for treating phantom limb pain. Cochrane Database Syst Rev 2016; 10: CD006380.

24. Wylde V, Dennis J, Beswick AD, Bruce J, Eccleston C, Howells N, et al. Systematic review of management of chronic pain after surgery. Br J Surg 2017; 104: 1293-1306.

25. Kumar V, Garg R, Bharati SJ, Gupta N, Bhatanagar S, Mishra S, et al. Long-term high-dose oral morphine in phantom limb pain with no addiction risk. Indian J Palliat Care 2015; 21: 85-87.

26. Robinson LR, Czerniecki JM, Ehde DM, Edwards WT, Judish DA, Goldberg ML, et al. Trial of amytryptyline for relief of pain in amputees: results of a randomized controlled study. Arch Phys Med Rehabil 2004; 85: 1-6.

27. Wilder-Smith $\mathrm{CH}$, Hill LT, Laurent S. Postamputation pain and sensory changes in treatment-naive patients: characteristics and responses to treatment with tramadol, amitriptyline, and placebo. Anesthesiology 2005; 103: 619-628.

28. Wang X, Yi Y, Tang D, Chen Y, Jiang Y, Peng J, et al. Gabapentin as an adjuvant therapy for prevention of acute phantom-limb pain in pediatric patients undergoing amputation for malignant bone tumors: a prospective double-blind randomized controlled trial. J Pain Symptom Manage 2018; 55: 721-727.

29. Wössner S, Weber K, Steinbeck AC, Oberhauser M, Feuerecker M. Pregabalin as adjunct in a multimodal pain therapy after traumatic foot amputation - A case report of a 4-year-old girl. Scand J Pain 2017; 17: 146-149.

30. Valproic Acid for the Prevention of Post-Amputation Pain. ClinicalTrials.gov Identifier: NCT01928849. Access: 14.04.2020.

31. Loy BM, Britt RB, Brown JN. Memantine for the treatment of phantom limb pain: a systematic review. J Pain Palliat Care Pharmacother 2016; 30: 276-283.

32. Yousef AA, Aborahma AM. The preventive value of epidural calcitonin in patients with lower limb amputation. Pain Med 2017; 18: 1745-1751.

33. Viana R, Payne MW. Use of calcitonin in recalcitrant phantom limb pain complicated by heterotopic ossification. Pain Res Manag 2015; 20: 229-233.

34. Mittal SO, Safarpour D, Jabbari B. Botulinum toxin treatment of neuropathic pain. Semin Neurol 2016; 36: 73-83.

35. Pasquina PF, Perry BN, Alphonso AL, Finn S, Fitzpatrick KF, Tsao JW. Residual limb hyperhidrosis and rimabotulinumtoxinB: a randomized placebo-controlled study. Arch Phys Med Rehabil 2016; 97: 659-664.

36. Münger M, Pinto CB, Pacheco-Barrios K, Duarte D, Enes Gunduz M, Simis M, et al. Protective and risk factors for phantom limb pain and residual limb pain severity. Pain Pract 2020; 20: 578-587.

37. Raichle KA, Osborne TL, Jensen MP, Ehde DM, Smith DG, Robinson LR. Preoperative state anxiety, acute postoperative pain, and analgesic use in persons undergoing lower limb amputation. Clin J Pain 2015; 31: 699-706.

38. Sahu A, Gupta R, Sagar S, Kumar M, Sagar R. A study of psychiatric comorbidity after traumatic limb amputation: a neglected entity. Ind Psychiatry J 2017; 26: 228-232.

39. Srivastava D. Chronic post-amputation pain: peri-operative management - review. Br J Pain 2017; 11: 192-202.

40. Pazzaglia M, Leemhuis E, Giannini AM, Haggard P. The homuncular jigsaw: investigations of phantom limb and body awareness following brachial plexus block or avulsion. J Clin Med 2019; 8: 182.

41. Takeuchi N, Izumi SI, Ota J, Ueda J. Neural plasticity on body representations: advancing translational rehabilitation. Neural Plast 2016; 2016: 9737569. 
42. Leemhuis E, De Gennaro L, Pazzaglia AM. Disconnected body representation: neuroplasticity following spinal cord injury. J Clin Med 2019; 8: 2144.

43. Harahap ZCS, Amin MM, Effendy E. Phantom pain limb: a case report in a soldier - theory and therapy. Open Access Maced J Med Sci 2019; 7: 2691-2694.

44. Baby S, Chaudhury S, Walia TS. Evaluation of treatment of psychiatric morbidity among limb amputees. Ind Psychiatry J 2018; 27: 240-248.

45. Hosseini SA, Padhy RK. Body Image Distortion. Treasure Island (FL): StatPearls Publishing; 2020.

46. Flahaut M, Laurent NL, Michetti M, Hirt-Burri N, Jensen W, Lontis R, et al. Patient care for postamputation pain and the complexity of therapies: living experiences. Pain Manag 2018; 8: 441-453.

47. Razmus M, Daniluk B, Markiewicz P. Phantom limb phenomenon as an example of body image distortion. Curr Probl Psychiatry 2017; 18: 153-159.

48. Fuchs X, Flor H, Bekrater-Bodmann R. Psychological factors associated with phantom limb pain: a review of recent findings. Pain Res Manag 2018; 2018: 5080123.

49. Durmus D, Safaz I, Adıgüzel E, Uran A, Sarısoy G, Goktepe AS, et al. The relationship between prosthesis use, phantom pain and psychiatric symptoms in male traumatic limb amputees. Compr Psychiatry 2015; 59: 45-53.

50. DeMoss P, Ramsey LH, Karlson CW. Phantom limb pain in pediatric oncology. Front Neurol 2018; 9: 219.

51. Ahuja V, Thapa D, Ghai B. Strategies for prevention of lower limb post-amputation pain: a clinical narrative review. J Anaesthesiol Clin Pharmacol 2018; 34: 439-449.

52. Hanyu-Deutmeyer AA, Cascella M, Varacallo M. Phantom Limb Pain. Treasure Island (FL): StatPearls Publishing; 2020. 